\title{
Colour Fields of the Static Pentaquark System Computed in SU(3) Lattice QCD
}

\author{
Nuno Cardoso* and Pedro Bicudo ${ }^{\dagger}$ \\ CFTP, Departamento de Física, Instituto Superior Técnico, \\ Universidade Técnica de Lisboa, Av. Rovisco Pais, 1049-001 Lisbon, Portugal
}

\begin{abstract}
We compute the colour fields of SU(3) lattice QCD created by static pentaquark systems, in a $24^{3} \times 48$ lattice at $\beta=6.2$ corresponding to a lattice spacing $a=0.07261(85) \mathrm{fm}$. We find that the pentaquark colour fields are well described by a multi-Y-type shaped flux tube. The flux tube junction points are compatible with Fermat-Steiner points minimizing the total flux tube length. We also compare the pentaquark flux tube profile with diquark-diantiquark central flux tube profile in the tetraquark and the quark-antiquark fundamental flux tube profile in the meson, and they match, thus showing that the pentaquark flux tubes are composed of fundamental flux tubes.
\end{abstract}

\section{INTRODUCTION}

Here we study the colour field flux tubes produced by static pentaquarks in SU(3) lattice QCD. Unlike the colour fields of simpler few-body systems, say mesons, baryons and hybrids, [1-4], the pentaquark fields have not been previously studied in lattice QCD. This study is relevant both for the solution of theoretical problems and for the development of phenomenological models of QCD.

Quark confinement remains one of the main open theoretical problems of particle physics. In lattice QCD, flux tubes composed of colour-electric and colour-magnetic fields have been observed and this constitutes a very important clue for the understanding of quark confinement. Since the onset of QCD with its asymptotic freedom and infrared slavery, it is well known that confinement is due to the gluon fields and suppressed by the quark fields. It is thus important to measure the different possible flux tubes of pure gauge lattice QCD, to provide data for any theoretical attempt to solve the QCD confinement problem.

Moreover, in what concerns phenomenology, the study of the colour fields in a pentaquark is important to discriminate between different multi-quark Hamiltonian models, quark models with two-body interactions only [5] as in the original quark model, from the string flip-flop model with a multi-body potential [6]. In the string flipflop model, the colour charges are connected by strings disposed geometrically in order to minimize the total string length. The strings constitute the limit of very thin elementary flux tubes. An elementary or fundamental flux tube is the flux tube connecting the quark and antiquark of a meson, where the quark is in the triplet or fundamental representation of QCD. For instance in the two quark two antiquark system, depending of the position of these colour charges, the minimal string may be a two-meson string, or a tetraquark string, shaped like a double-Y flux tube, as in Fig. 1, composed of five linear fundamental flux tubes meeting in two Fermat-Steiner points [7-9]. A Fermat, or Steiner, point is defined as a
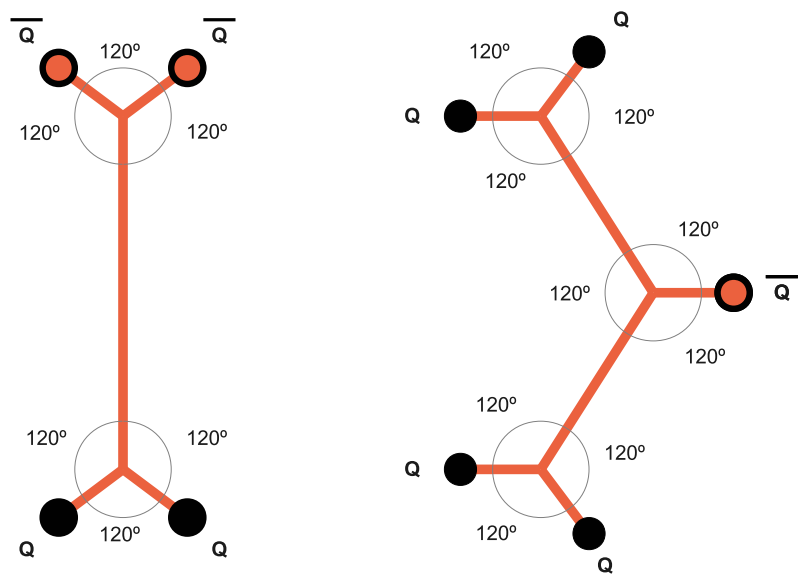

Figure 1: (Colour online.) In the string flip-flop model, thin elementary flux tubes similar to strings connect the colour charges in order to minimize the total length of

the strings. Whenever geometrically possible, three elementary flux tubes meet in a Fermat-Steiner point at an angle of $\alpha=120^{\circ}$. Here we depict planar examples of a tetraquark and a pentaquark flux tubes.

junction minimizing the total length of strings, where linear individual strings join at $120^{\circ}$ angles. When the positions of the colour charges change, the potential may thus flip from one four-body potential to a pair of two-body potentials and flop back again. Notice the flip flop potential, compatible with the confining component of the flux tubes explored here, lead to tetraquark boundstates, below the strong decay threshold to pairs of mesons [7, 1012]. Recent investigations found that, even above the strong decay threshold, the presence of a centrifugal barrier in high angular momentum multiquarks may increase the stability of the system $[6,13]$. The multiquark hamiltonians are important to understand not only the elusive multiquark hadrons, but also high density QCD where many quarks may overlap.

Experimentally, multiquark exotic hadrons have been searched for many years because as soon as the quarks were proposed in the sixties to classify the meson and 


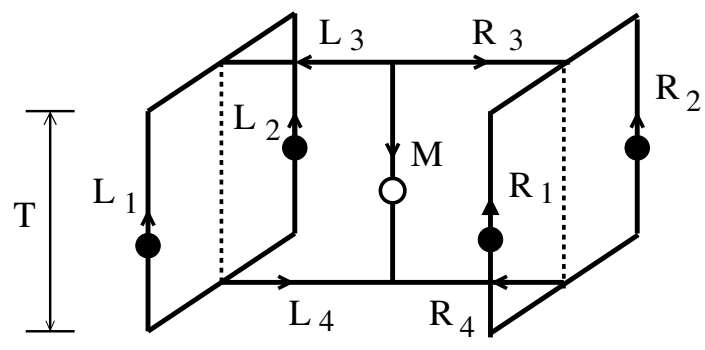

Figure 2: Pentaquark Wilson loop as defined by Okiharu et al. [15, 16]. Here we extend this Wilson loop with different paths $L_{i}$ and $R_{i}$ for the quarks and $M_{i}$ for the antiquark.

baryon resonances, and the quark model was proposed in the seventies [14], it became clear that systems with more than three quarks could also possibly exist. One of the main problems of hadronic physics is thus to determine whether multiquark resonances exist or not, and weather the possible multiquark resonances are narrow or wide.

The simplest multiquark system is the tetraquark, and it was already proposed by Jaffe in the seventies [17] as a bound state formed by two quarks and two antiquarks. Presently some observed resonances are tetraquark candidates. Very recently the Belle Collaboration made the tantalizing observation [18], in five different $\Upsilon(5 \mathrm{~S})$ decay channels of two new charged bottomonium resonances $\mathrm{Z}_{b}$ with masses of $10610 \mathrm{MeV} / \mathrm{c}^{2}$ and $10650 \mathrm{MeV} / \mathrm{c}^{2}$ and narrow widths of the order or $15 \mathrm{MeV}$. Since all standard bottomonia are neutrally charged, these two new resonances have a flavour only compatible with $b \bar{b} u \bar{d}$ tetraquarks. In 2003, the $\mathrm{X}(3872)$ observed by the Bell Collaboration $[19,20]$ was suggested as a tetraquark candidate by Maiani et al [21]. In 2004, the $\mathrm{D}_{\mathrm{sJ}}(2632)$ state seen in Fermilab's SELEX [22, 23] was suggested as a possible tetraquark candidate. In 2009, Fermilab announced the discovery of $\mathrm{Y}(4140)$, which may also be a tetraquark [24]. There are as well indications that the Y(4660) could be a tetraquark state [25]. The $\Upsilon(5 \mathrm{~S})$ bottomonium has also been recently suggested to be a tetraquark resonance [26]. However a better understanding of tetraquarks is necessary to confirm or disprove the $\mathrm{X}, \mathrm{Y}, \mathrm{Z}$ and possibly also other light resonances candidates as tetraquark states.

The pentaquark is the next in the multiquark hadron series. Pentaquark hadrons were already proposed in the eighties by Manohar [27] and Chemtob [28], inspired by extensions of the Skyrme model. In the 2000s a burst of interest was sparkled by a discovery claim of the $\Theta$ pentaquark by Nakano et al [29]. This led to many experimental and lattice QCD studies of pentaquarks, together with hundreds of theoretical estimations of the $\Theta$ properties. However the resonance $\Theta$ ended up by not being confirmed by the scientific community [30, 31]. The many hundreds of publications on the subject, with disparate

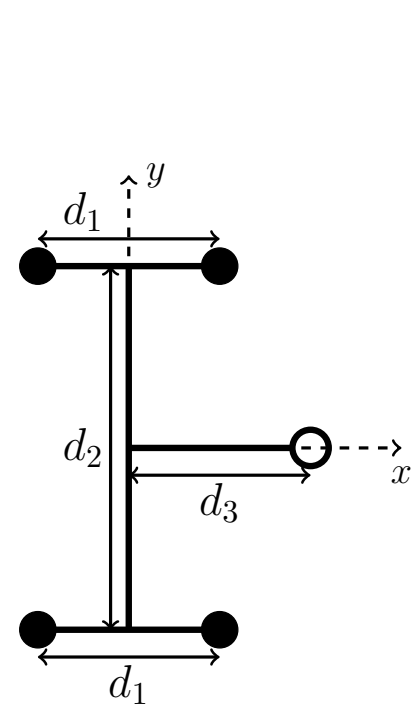

(a)

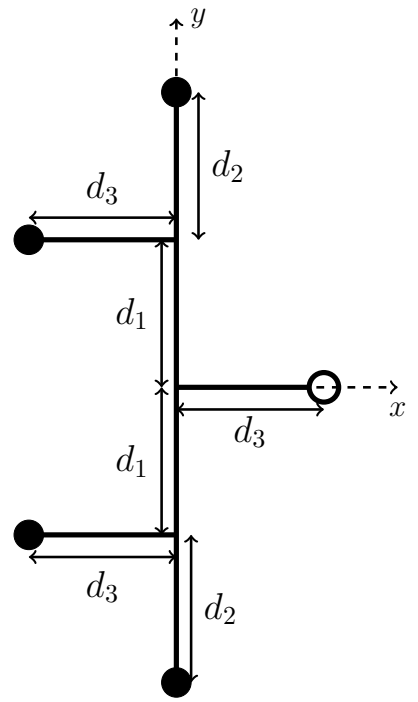

(b)
Figure 3: Projection in the spacial dimensions of the different Wilson loop geometries for the static pentaquark studied in this work. The solid dots correspond to the quarks positions and the open dots to the antiquarks. The solid lines correspond to the space-like Wilson paths.

conclusions, show that the $\Theta$ pentaquark was beyond the scope of the scientific techniques utilized in the 2000s.

The multiquark hadrons are thus very elusive systems, much harder to observe experimentally, to understand in models, and to simulate in lattice QCD than the conventional mesons and baryons. Nevertheless, inasmuch as the understanding of confinement, the existence/nonexistence of multiquark hadrons remain an important problem in $\mathrm{QCD}$, to be further explored in the future PANDA experiment at GSI.

It is thus important to proceed with the well defined program of understanding the static potentials and flux tubes of multiquarks in quenched Lattice QCD.

In the last years, the static tetraquark potential has been studied in Lattice QCD computations [3234]. The authors concluded that when the quark-quark are well separated from the antiquark-antiquark, the tetraquark potential is consistent with One Gluon Exchange Coulomb potentials plus a four-body confining potential, suggesting the formation of a double-Y flux tube, typical of the four-body potential of the string flipflop model as in Fig. 1, composed of five linear fundamental flux tubes meeting in two Fermat-Steiner points [7-9]. This flux tube geometry was confirmed by Lattice QCD studies of the flux tubes produced by a static tetraquark system $[35,36]$. In what concerns the pentaquark, static potentials have already been explored in a geometry with the antiquark situated in the centre of 
Table I: Pentaquark geometries studied and number of lattice configurations used in this work. The geometry type is outlined in Fig. 3. The column Id corresponds to the numbering used in the text.

\begin{tabular}{cccccc}
\hline \hline Id & Geometry Type & $\mathbf{d}_{\mathbf{1}}$ & $\mathbf{d}_{\mathbf{2}}$ & $\mathbf{d}_{\mathbf{3}}$ & \# Configs. \\
\hline (i) & Fig. 3a & 8 & 8 & 0 & 551 \\
(ii) & Fig. 3a & 8 & 8 & 6 & 549 \\
(iii) & Fig. 3b & 4 & 4 & 8 & 544 \\
(iv) & Fig. 3b & 6 & 4 & 8 & 1121 \\
\hline \hline
\end{tabular}

the four quarks [15, 32], also consistent with a string flipflop model, in this case with only six fundamental flux tubes and two Fermat-Steiner points.

Here we proceed with the flux tube research program, studying the flux tubes of static pentaquarks in pure gauge SU(3) lattice QCD. In Section II we detail the framework we set to measure the flux tubes. We also extend the geometries explored in the static potential studies. In Section III we expose our results and conclude.

\section{SIMULATING THE PENTAQUARK FLUX TUBES IN LATTICE QCD}

The static potential for the pentaquark was already studied in the lattice QCD by [32] and [15, 16] utilizing generalized Wilson loops. Here we use similar Wilson loops to place a static system of four quarks and one antiquark in the lattice, in four different geometries. Moreover we measure the colour-electric and colour-magnetic fields produced by the static charges.

The Wilson loop operator for the pentaquark system is defined in a gauge-invariant way, as illustrated in Fig. 2 , by

$$
W_{5 Q}=\frac{1}{3 !} \epsilon^{i j k} \epsilon^{i^{\prime} j^{\prime} k^{\prime}} M^{i i^{\prime}}\left(R_{3} R_{12} R_{4}\right)^{j j^{\prime}}\left(L_{3} L_{12} L_{4}\right)^{k k^{\prime}}
$$

where

$$
\begin{aligned}
& R_{12}^{i^{\prime} i}=\frac{1}{2} \epsilon^{i j k} \epsilon^{i^{\prime} j^{\prime} k^{\prime}} R_{1}^{j j^{\prime}} R_{2}^{k k^{\prime}}, \\
& L_{12}^{i^{\prime} i}=\frac{1}{2} \epsilon^{i j k} \epsilon^{i^{\prime} j^{\prime} k^{\prime}} L_{1}^{j j^{\prime}} L_{2}^{k k^{\prime}} .
\end{aligned}
$$

The projection in the spacial dimensions of our four different Wilson loop geometries for the static pentaquark is illustrated in Fig. 3. The distances of the geometries are quantified in Table I. The labelling of the geometries together with the number of lattice configurations used in this work are also shown in Table I.

We compute the colour electric and the colour magnetic fields, by using the correlators of the plaquettes $P_{\mu \nu}$ and the Wilson loop $W_{5 Q}$. We define the plaquettes as $P_{\mu \nu}=1-\frac{1}{3} \operatorname{Tr}\left[U_{\mu}(\mathbf{s}) U_{\nu}(\mathbf{s}+\boldsymbol{\mu}) U_{\mu}^{\dagger}(\mathbf{s}+\boldsymbol{\nu}) U_{\nu}^{\dagger}(\mathbf{s})\right]$.
Table II: Fermat-Steiner points for the pentaquark geometries studied. The geometry type is outlined in Fig. 3 and Table I.

\begin{tabular}{cccc}
\hline \hline \multicolumn{3}{c}{ Fermat-Steiner Points } \\
Id & $\mathbf{r}_{\mathbf{I}}$ & $\mathbf{r}_{\mathbf{I I}}$ & $\mathbf{r}_{\mathbf{I I I}}$ \\
\hline (i) & $(0,-1.691,0)$ & $(0,1.691,0)$ & $(0,0,0)$ \\
(ii) & $(3.897,-3.830,0)$ & $(3.897,3.830,0)$ & $(6,0,0)$ \\
(iii) & $(-2.309,-4,0)$ & $(-2.309,4,0)$ & $(0,0,0)$ \\
(iv) & $(-2.309,-6,0)$ & $(-2.309,6,0)$ & $(1.155,0,0)$ \\
\hline \hline
\end{tabular}

With this definition, the chromofields are given by

$$
\begin{aligned}
\left\langle E_{i}^{2}\right\rangle & =\left\langle P_{0 i}\right\rangle-\frac{\left\langle W_{5 Q} P_{0 i}\right\rangle}{\left\langle W_{5 Q}\right\rangle} \\
\left\langle B_{i}^{2}\right\rangle & =\frac{\left\langle W_{5 Q} P_{j k}\right\rangle}{\left\langle W_{5 Q}\right\rangle}-\left\langle P_{j k}\right\rangle,
\end{aligned}
$$

with the indices $j$ and $k$ complementing index $i$. The lagrangian and energy densities are given by $\mathcal{L}=\frac{1}{2}\left(E^{2}-\right.$ $\left.B^{2}\right)$ and $\mathcal{H}=\frac{1}{2}\left(E^{2}+B^{2}\right)$.

To compute the static field expectation value, we plot the expectation value $\left\langle E_{i}^{2}(\mathbf{r})\right\rangle$ or $\left\langle B_{i}^{2}(\mathbf{r})\right\rangle$ as a function of the temporal extension $T$ of the Wilson loop. At sufficiently large $T$, the groundstate corresponding to the studied quantum numbers dominates, and the expectation value tends to a horizontal plateau. In order to improve the signal to noise ratio of the Wilson loop, we use 50 iterations of APE Smearing with $w=0.2$ (as in $[3,35])$ in the spatial directions and one iteration of hypercubic blocking (HYP) in the temporal direction, [37], with $\alpha_{1}=0.75, \alpha_{2}=0.6$ and $\alpha_{3}=0.3$. Note that these two procedures are only applied to the Wilson Loop, not to the plaquette.

To check if the pentaquark flux tube produces a clear signal, we study the $\chi^{2} /$ dof of our pentaquark $T$ plateaux. But, surprisingly, event at some of the distances illustrated in Fig. 4, where the string flip-flop potential would favour the meson-baryon flux tube, with a lower energy than the pentaquark flux tube, we find $T$ plateaux with a good $\chi^{2} /$ dof. This shows that the mixing between the pentaquark flux tube and the mesonbaryon flux tube is small, and it is possible to study clear pentaquark flux tubes even at relatively large diquark distances.

To compute the fields, we fit the horizontal plateaux obtained for each point $\mathbf{r}$ determined by the plaquette position, but we consider $z=0$ for simplicity. We finally compute the error bars of the fields with the jackknife method.

We compute the Fermat-Steiner points with the iterative method of Bicudo et al. [8]. We have five quarks(antiquarks) with the label $i$ and three Fermat- 

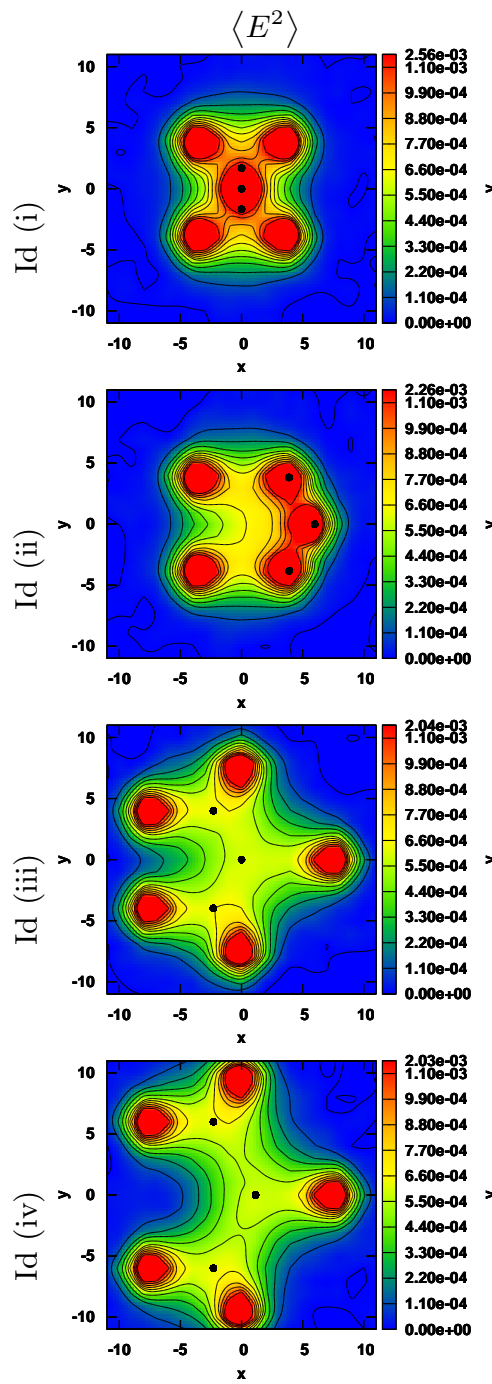
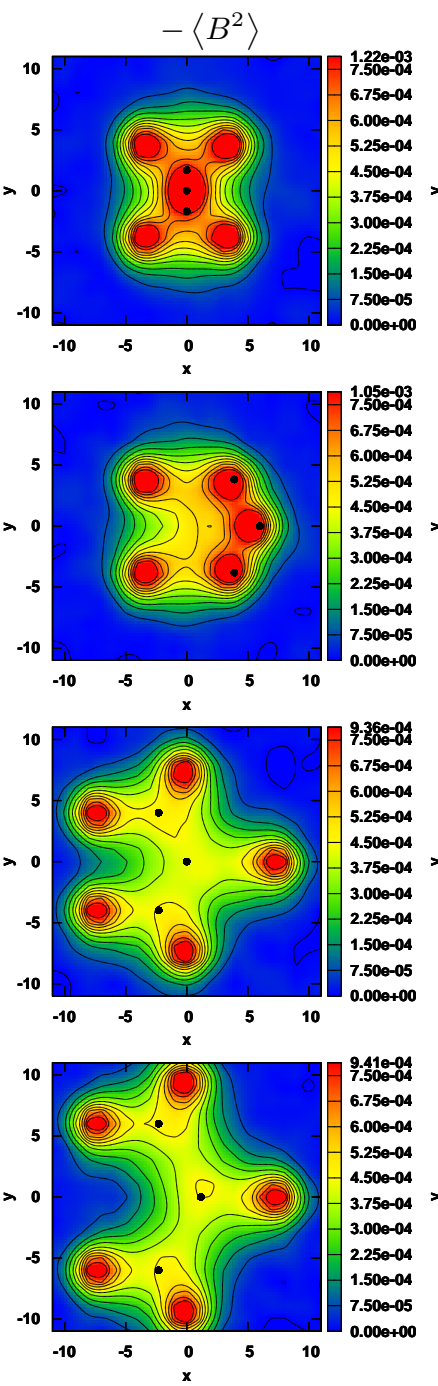
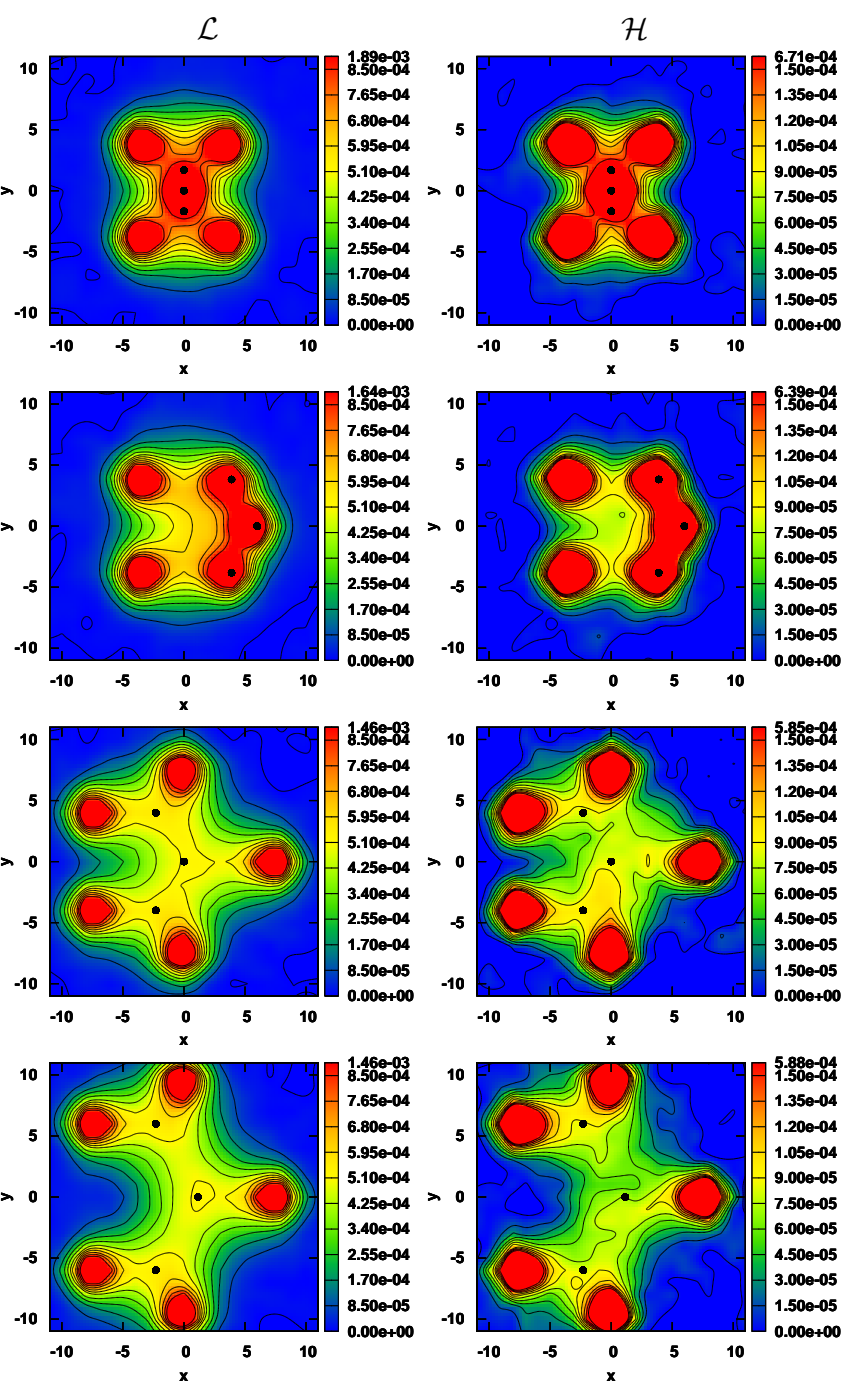

Figure 4: (Colour online.) Density plots of the chromoelectric and chromomagnetic fields and Lagrangian and energy densities for the geometries defined in Table I. The black dot points correspond to the Fermat-Steiner points,

Table II. The results are presented in lattice spacing units.

Steiner points with label $a=I, I I, I I I$,

$$
\begin{aligned}
\mathbf{r}_{i} & =\left(x_{i}, y_{i}, z_{i}\right) \\
\mathbf{r}_{a} & =\left(x_{a}, y_{a}, z_{a}\right), \\
r_{i a} & =\sqrt{\left(x_{a}-x_{i}\right)^{2}+\left(y_{a}-y_{i}\right)^{2}+\left(z_{a}-z_{i}\right)^{2}} .
\end{aligned}
$$

To minimize the total length of the strings,

$$
d=r_{1 I}+r_{2 I}+r_{3 I I}+r_{4 I I}+r_{\overline{5} I I I}+r_{I I I I}+r_{I I I I I},
$$

we only need to solve one non-linear vector equation per
Fermat-Steiner point,

$$
\begin{aligned}
\mathbf{r}_{I} & =\frac{\frac{\mathbf{r}_{1}}{r_{1 I}}+\frac{\mathbf{r}_{2}}{r_{2 I}}+\frac{\mathbf{r}_{I I I}}{r_{I I I}}}{\frac{1}{r_{1 I}}+\frac{1}{r_{2 I}}+\frac{1}{r_{I I I}}}, \\
\mathbf{r}_{I I} & =\frac{\frac{\mathbf{r}_{3}}{r_{3 I I}}+\frac{\mathbf{r}_{4}}{r_{4 I I}}+\frac{\mathbf{r}_{I I I}}{r_{I I I I I}}}{\frac{1}{r_{3 I I}}+\frac{1}{r_{4 I I}}+\frac{1}{r_{I I I I I}}}, \\
\mathbf{r}_{I I I} & =\frac{\frac{\mathbf{r}_{I}}{r_{I I I I}}+\frac{\mathbf{r}_{I I}}{r_{I I I I I}}+\frac{\mathbf{r}_{5}}{r_{5 I I I}}}{\frac{1}{r_{I I I I}}+\frac{1}{r_{I I I I I}}+\frac{1}{r_{5} I I I}}
\end{aligned}
$$

\section{RESULTS AND CONCLUSION}

We remark that the signal is clear only if the paths considered in the Wilson loop overlap the flux tube. Thus we consider geometries for the Wilson loop where the paths 


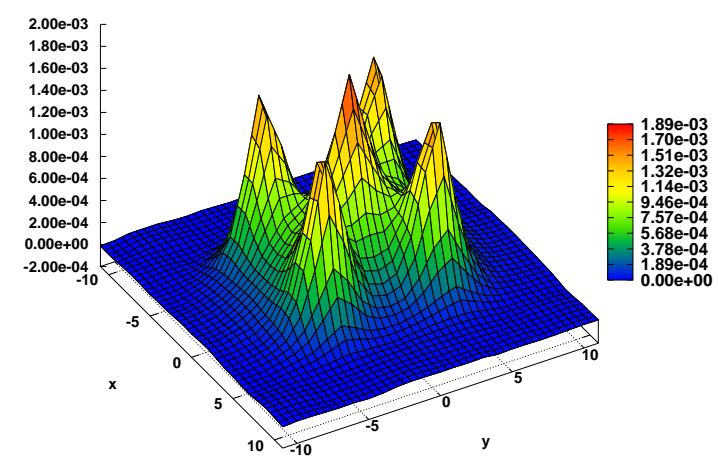

(a) Id (i)

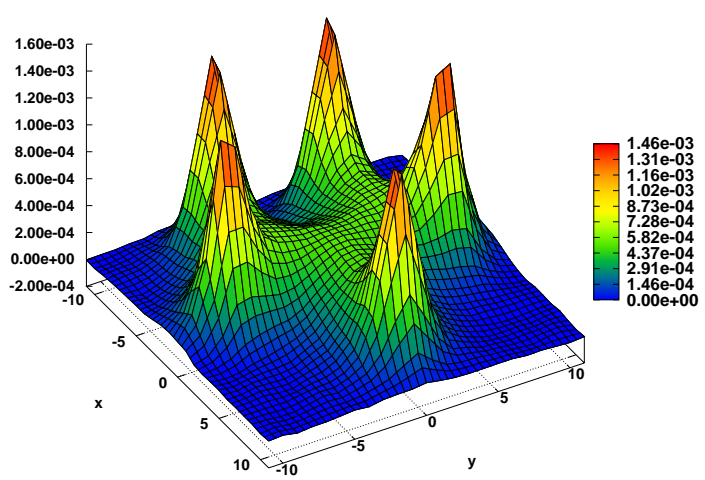

(c) Id (iii)

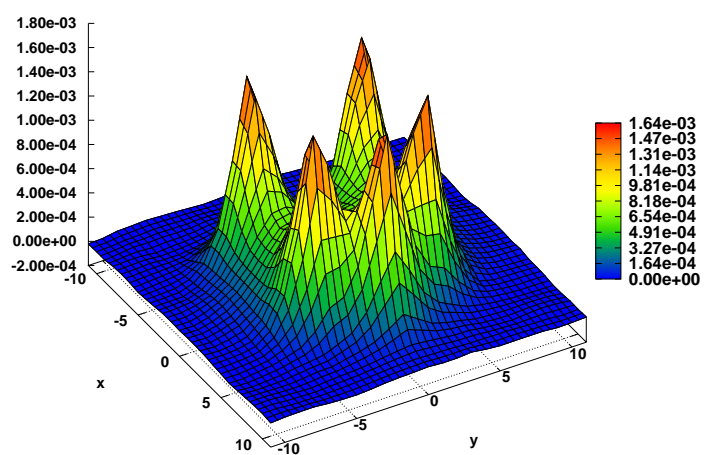

(b) Id (ii)

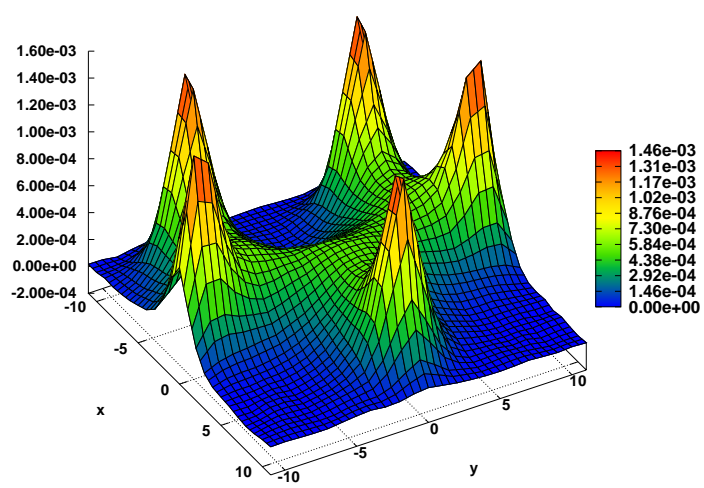

(d) Id (iv)

Figure 5: (Colour online) we show three-dimensional plots of the Lagrangian density for the geometries defined in Table I. The density enhancement is maximal in the location of the colour charges, and if the colour charges were close the Coulomb potential would be important. With our geometries the colour charges are separated, and the fundamental flux tubes connecting the charges and the Fermat-Steiner points are evident. The results are presented in lattice spacing units.

are just some lattice spacings distant from the expected string position in the string flip-flop model. We consider the four different Wilson loop geometries, detailed in Fig. 3 and in Table I. We only utilize planar geometries for the colour sources, in order to produce clearer pictures of the fields. The results for the colour field densities are presented only for the $x y$ plane since the colour sources are in this plane and the results with $z \neq 0$ are less interesting for this study. Then with colour field densities as a function of $x$ and $y$ we produce density plots and three-dimensional plots.

To produce the results presented in this work, we utilize quenched configurations in a $24^{3} \times 48$ lattice at $\beta=6.2$. The number of configurations used is described in Table I. We present our results in lattice spacing units of $a$, with $a=0.07261(85) \mathrm{fm}$ or $a^{-1}=2718 \pm 32$ $\mathrm{MeV}$. We generate our configurations in NVIDIA GPUs of the FERMI series (480, 580 and Tesla C2070) with a SU(3) CUDA code upgraded from our SU(2) combination of Cabibbo-Marinari pseudoheatbath and overrelaxation algorithm [38-40]. Our SU(3) updates involve three $\mathrm{SU}(2)$ subgroups, we work with 9 complex numbers, and we reunitarize the matrix.

The results for the colour fields, the energy and lagrangian densities are shown in Figs. 4-5. The figures clearly exhibit multi-Y-type shaped flux tubes. We also plot the Fermat-Steiner points defined in Table II. The Fermat-Steiner points of geometries (i) and (ii) are of different type from the Fermat-Steiner points of geometries (iii) and (iv), since in the first geometries angles of $120^{\circ}$ between the fundamental strings are not possible and thus the central Fermat-Steiner point has merged with the antiquark source. Nevertheless, and although the flux tubes have a finite width and are not infinitely thin as is assumed in the string flip-flop models, and although the Coulomb component of the potential is certainly important, we notice the junctions for the elementary flux tubes are clearly close to the computed Fermat-Steiner points. This validates the use of string flip-flop models for the quark confinement in constituent quark models.

In Fig. 6, we compare the chromoelectric field profile for the pentaquark, tetraquark and the quark-antiquark system in the middle of the flux tube. The tetraquark and the quark-antiquark results were obtained by [35]. 


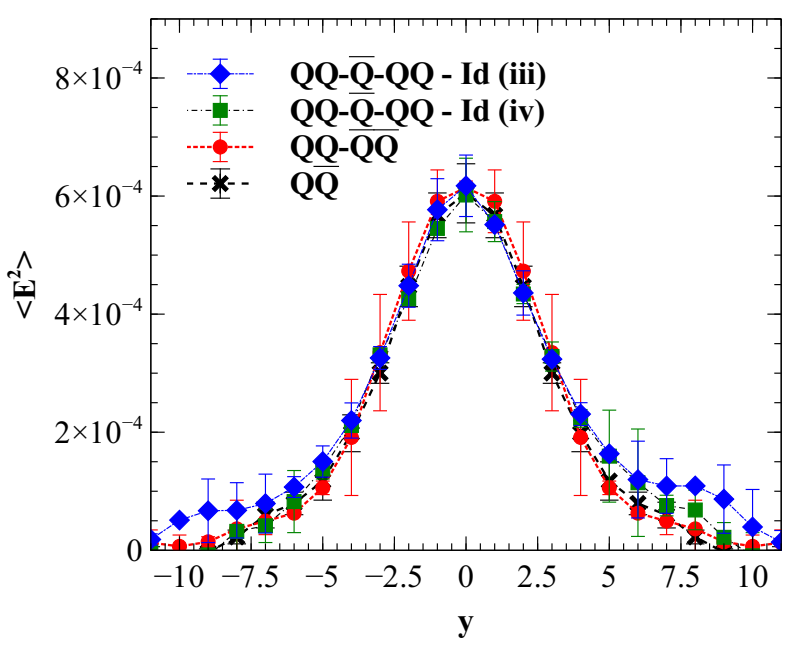

Figure 6: (Colour online.) Profile of the chromoelectric field for the pentaquark, tetraquark and quark-antiquark systems. The pentaquark profile corresponds to the geometry profile outlined in Fig. 3 and Table I along $x=4$. The tetraquark and quark-antiquark results are from [35], in the middle of the flux tube.

The three chromoelectric fields are identical up to the error bars. This confirms that the pentaquark flux tube is composed of a set of fundamental flux tubes with Fermat-Steiner junctions, and again validates the string flip-flop models as models for the quark confinement in constituent quark models.

Multiquark stability is a subtle theoretical problem, requiring the correct understanding and calibration of the quark interactions. Combining our pentaquark results with the flux tube studies of mesons [41], baryons [42], hybrids [3], glueballs [43], and tetraquarks [35, 36] we finally feel confident that the string flip-flop potential, where fundamental strings with the minimal possible length link the static colour sources, is the correct phenomenological model for the confinement of any system of static quarks, antiquarks and gluons. Whether the string flip-flop confining potential together with a correct short-range potential lead to multiquark narrow resonances or boundstates remains a difficult quantum mechanical problem, but very interesting to the confinement and quark model experts.

This work was partly funded by the FCT contracts, POCI/FP/81933/2007, CERN/FP/83582/2008, PTDC/FIS/100968/2008, CERN/FP/109327/2009, CERN/FP/116383/2010 and CERN/FP/123612/2011. Nuno Cardoso is also supported by FCT under the contract SFRH/BD/44416/2008.
* Electronic address: nuno.cardoso@ist.utl.pt

$\dagger$ Electronic address: bicudo@ist.utl.pt

[1] H. Ichie, V. Bornyakov, T. Streuer, and G. Schierholz, Nucl. Phys. A721, 899 (2003), arXiv:hep-lat/0212036 .

[2] F. Okiharu and R. M. Woloshyn, Eur. Phys. J. C35, 537 (2004), arXiv:hep-lat/0402009 .

[3] M. Cardoso, N. Cardoso, and P. Bicudo, Phys. Rev. D81, 034504 (2010), arXiv:0912.3181 [hep-lat] .

[4] N. Cardoso, M. Cardoso, and P. Bicudo, (2010), arXiv:1004.0166 [hep-lat] .

[5] I. J. General, P. Wang, S. R. Cotanch, and F. J. LlanesEstrada, Phys.Lett. B653, 216 (2007), arXiv:0707.1286 [hep-ph] .

[6] P. Bicudo and M. Cardoso, (2010), arXiv:1010.0281 [hep$\mathrm{ph}]$.

[7] J. Vijande, A. Valcarce, and J.-M. Richard, Phys.Rev. D76, 114013 (2007), arXiv:0707.3996 [hep-ph] .

[8] P. Bicudo and M. Cardoso, Phys.Lett. B674, 98 (2009), arXiv:0812.0777 [physics.comp-ph] .

[9] J.-M. Richard, (2009), arXiv:0905.2308 [hep-ph] .

[10] M. W. Beinker, B. C. Metsch, and H. R. Petry, J. Phys. G22, 1151 (1996), arXiv:hep-ph/9505215 .

[11] S. Zouzou, B. Silvestre-Brac, C. Gignoux, and J. M. Richard, Z. Phys. C30, 457 (1986).

[12] B. A. Gelman and S. Nussinov, Phys. Lett. B551, 296 (2003), arXiv:hep-ph/0209095 .

[13] M. Karliner and H. J. Lipkin, Phys. Lett. B575, 249 (2003), arXiv:hep-ph/0402260 .

[14] A. De Rujula, H. Georgi, and S. Glashow, Phys.Rev. D12, 147 (1975).

[15] F. Okiharu, H. Suganuma, and T. T. Takahashi, Phys.Rev.Lett. 94, 192001 (2005), arXiv:heplat/0407001 [hep-lat] .

[16] F. Okiharu et al., (2005), arXiv:hep-ph/0507187 .

[17] R. L. Jaffe, Phys. Rev. D15, 267 (1977).

[18] I. Adachi (Belle Collaboration), (2011), arXiv:1105.4583 [hep-ex] .

[19] S. K. Choi et al. (Belle), Phys. Rev. Lett. 91, 262001 (2003), arXiv:hep-ex/0309032 .

[20] D. E. Acosta et al. (CDF II), Phys. Rev. Lett. 93, 072001 (2004), arXiv:hep-ex/0312021.

[21] L. Maiani, F. Piccinini, A. D. Polosa, and V. Riquer, Phys. Rev. D71, 014028 (2005), arXiv:hep-ph/0412098 .

[22] S. Y. Jun (the SELEX), Prepared for Flavor Physics and CP Violation (FPCP 2004), Daegu, Korea, 4-9 Oct 2004.

[23] P. S. Cooper, J. Phys. Conf. Ser. 9, 53 (2005).

[24] N. Mahajan, Phys. Lett. B679, 228 (2009), arXiv:0903.3107 [hep-ph] .

[25] G. Cotugno, R. Faccini, A. D. Polosa, and C. Sabelli, Phys. Rev. Lett. 104, 132005 (2010), arXiv:0911.2178 [hep-ph] .

[26] A. Ali, C. Hambrock, and M. J. Aslam, Phys. Rev. Lett. 104, 162001 (2010), arXiv:0912.5016 [hep-ph] .

[27] A. V. Manohar, Nucl.Phys. B248, 19 (1984).

[28] M. Chemtob, Nucl.Phys. B256, 600 (1985).

[29] T. Nakano et al. (LEPS Collaboration), Phys.Rev.Lett. 91, 012002 (2003), arXiv:hep-ex/0301020 [hep-ex] .

[30] A. R. Dzierba, C. A. Meyer, and A. P. Szczepaniak, J.Phys.Conf.Ser. 9, 192 (2005), arXiv:hep-ex/0412077 [hep-ex] .

[31] A. Martinez Torres and E. Oset, Phys.Rev.Lett. 105, 
092001 (2010), arXiv:1008.4978 [nucl-th] .

[32] C. Alexandrou and G. Koutsou, Phys. Rev. D71, 014504 (2005), arXiv:hep-lat/0407005 .

[33] F. Okiharu, H. Suganuma, and T. T. Takahashi, Phys. Rev. D72, 014505 (2005), arXiv:hep-lat/0412012.

[34] V. Bornyakov, P. Boyko, M. Chernodub, and M. Polikarpov, (2005), arXiv:hep-lat/0508006 [hep-lat] .

[35] N. Cardoso, M. Cardoso, and P. Bicudo, Phys.Rev. D84, 054508 (2011), arXiv:1107.1355 [hep-lat] .

[36] M. Cardoso, N. Cardoso, and P. Bicudo, Phys.Rev. D86, 014503 (2012), arXiv:1204.5131 [hep-lat] .

[37] A. Hasenfratz and F. Knechtli, Phys. Rev. D 64-3, 034504 (2001).
[38] N. Cardoso and P. Bicudo, J.Comput.Phys. 230, 3998 (2011), arXiv:1010.4834 [hep-lat] .

[39] N. Cardoso and P. Bicudo, (2011), arXiv:1112.4533 [heplat] .

[40] (2011), the CUDA codes are available at Portuguese Lattice QCD collaboration, http://nemea.ist.utl.pt/ ptqcd.

[41] G. S. Bali, K. Schilling, and C. Schlichter, Phys. Rev. D51, 5165 (1995), arXiv:hep-lat/9409005 .

[42] T. T. Takahashi, H. Suganuma, and H. Ichie, (2004).

[43] M. Cardoso and P. Bicudo, Phys.Rev. D78, 074508 (2008), arXiv:0807.1621 [hep-lat] . 